\title{
Rapid and clinically significant response to masitinib in the treatment of mucosal primary esophageal melanoma with somatic KIT exon 11 mutation involving brain metastases: A case report
}

\author{
Jarmila Prosvicova a , Sarka Lukesova ${ }^{a, b}$, Jindrich Kopeckyc, Jiri Grim', Zdenek Papik ${ }^{d}$, Renata Kolarovad, Blanka Navratilovae \\ Patrice Dubreuil ${ }^{\mathrm{fg}}$, Julie Agopian ${ }^{g}$, Colin Mansfield ${ }^{g}$, Alan Moussy ${ }^{g}$, Olivier Hermine ${ }^{g, h}$
}

\begin{abstract}
Background. Malignant melanoma in the gastrointestinal tract may be primary or metastatic. Mucosal melanoma is a quite rare and aggressive disease, growing hidden and diagnosed with a certain delay which makes treatment difficult. Case Report. The authors present the first patient with c-kit exon 11 mutated primary esophageal melanoma treated with oral tyrosine kinase inhibitor masitinib. A 55-year-old-man presented with esophageal melanoma metastising into visceral organs and to the brain. The patient showed objective and clinical significant therapeutic response to masitinib. After initiation of masitinib, dysphagia and odynophagia disappeared within 1 week. Following 1 month of treatment, computed tomography showed a regression in the number and size of brain metastatic lesions and regression in visceral lesions. This therapeutic response, despite the aggressive disease on treatment initiation, effectively enabled the patient to have 6 months of quality life.

Conclusion. This report corroborates the plausibility of treating advanced melanoma carrying a mutation of KIT with masitinib. It also raises the question of masitinib treatment beyond progression. Additionally, the observed masitinib treatment effect on the brain suggests accumulation of therapeutically relevant concentration of masitinib in the central nervous system. This observation has possible ramifications for treatment of intracranial neoplasms.
\end{abstract}

Key words: esophageal melanoma, masitinib, targeted therapy

Received: October 12, 2015; Accepted with revision: November 24, 2015; Available online: November 27, 2015 http://dx.doi.org/10.5507/bp.2015.061

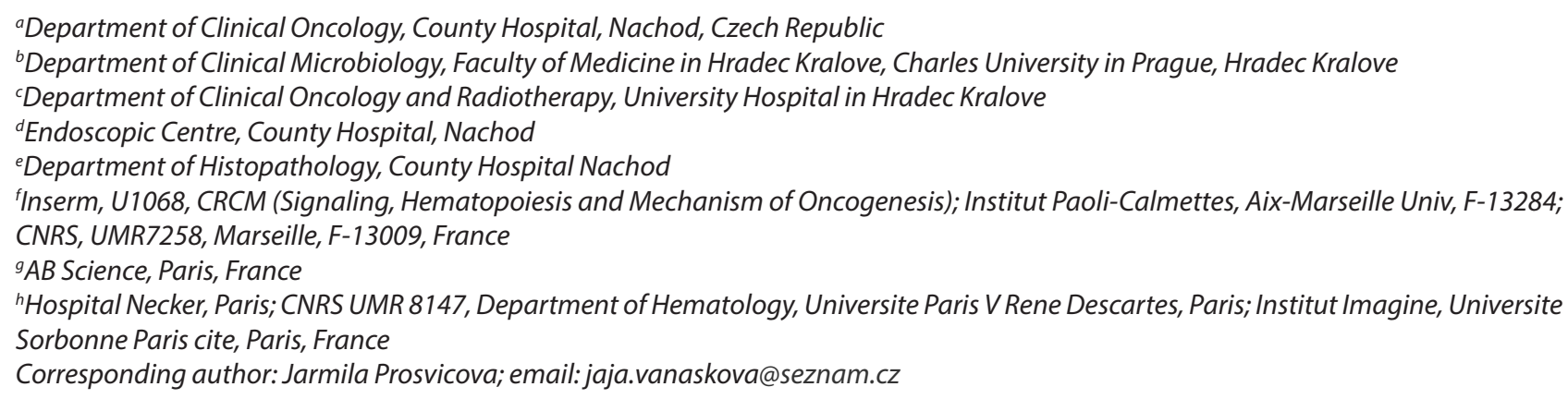

\section{INTRODUCTION}

Mucosal melanoma is a group of rare and aggressive disease, with mean survival rate of $2.2 \%$ at 5 years and a median survival rate of 10 months $^{1}$. Primary esophageal melanomas are even rarer, accounting for less than $1 \%$ of all esophageal tumors of which only $10 \%$ are primary melanoma. Primary esophageal melanoma comprises less than $0.05 \%$ of all melanoma subtypes ${ }^{2,3}$.

KIT is a transmembrane receptor tyrosine kinase, normally expressed on melanocytes. Mutations and amplification of KIT have been identified in melanomas arising from mucosal, acral or chronically sun-damaged surface. The alterations are usually mutually exclusive of BRAF and NRAS mutations.

\section{CASE REPORT}

A 55-year-old-man presented dysphagia worsening over the course of several weeks. Gastroscopy revealed endoluminal prominent exulcerated almost circular tumor in 30-41 cm from incisors, fragile, with contact bleeding (Fig. 1). Differential diagnosis of endoscopist was primary stromal tumor or mediastinal tumor protruding into esophagus. Primary esophageal melanoma was not considered during gastroscopy because of its rarity.

The conclusion from the histopathology was malignant melanoma of the esophagus, with atypical junction proliferation making primary esophageal melanoma more probable. PET/CT revealed thickening of the middle esophagus with metastatic involvement of para-esophageal and para-aortal lymph nodes, lungs, liver, bones, and soft 
tissues. The patient had had no personal history or signs of cutaneous or ocular melanoma.

Mutation analysis was performed for status of $K I T$ juxtamembrane mutation exon 9 and 11 and eligibility to enroll onto trial AB08026. Baseline radiologic assessments revealed aggressive melanoma, disease progression as compared with the assessments conducted just 1 month prior was obvious, particularly in axillar lymphadenopathy, left side fluidothorax, lung and pleural metastases, lymphadenopathy in mediastinum, liver metastasis, vertebral lesions in Th 11 ( $28 \times 20 \mathrm{~mm})$ and Th 7 (12 x $10 \mathrm{~mm}$ ), and soft tissue knots throughout trunk, breast muscles, and shoulders. Computed tomography also showed brain involvement with multiple lesions.

KIT exon 11 mutation was positive. The patient therefore started treatment with masitinib at a dose of $7.5 \mathrm{mg} /$ $\mathrm{kg} /$ day administered orally twice daily. At this time the patient was in good general health condition, actively working as a locksmith, although he was suffering from dysphagia and odynophagia. Just days after treatment initiation, the patient noticed symptomatic improvement and within 1 week he no longer experienced any difficulty in swallowing. Soft tissue knots, he had been able to touch in his muscles, disappeared. Radiologic assessment after 1 month of treatment showed marked response, reduction in size of axillar and mediastinal lymphadenopathy, reduced pleural and liver metastases, metastases in the lungs were no longer seen, thickness of the esophagus had reduced, and knots in soft tissues of the trunk had disappeared. Surprisingly, brain lesions were also in regression, by over $50 \%$ with respect to baseline. The patient's general health condition remained very good.

The next tumor assessment after another 2 months revealed progression with a number of new brain lesions. Otherwise, the marked response to masitinib still remained. Lungs remained in total remission; axillar lymphadenopathy, mediastinal lymphadenopathy, and knots in soft tissues of the trunk all remained in regression; liver lesions remained stable. Administration of masitinib was nevertheless stopped as per protocol rules on progressive disease and the patient underwent whole brain irradiation and palliative fotemustine. Brain MRI performed after 2 months showed tens of lesions. There was rapid regrowth of tumor lesions after withdrawal of masitinib. The patient was in very good general health condition and was able to work actively until he died very probable of bleeding in brain metastases.

\section{DISCUSSION}

Mucosal melanoma is a rare disease, accounting for $1.3 \%$ of all melanomas ${ }^{4}$, and is typically diagnosed at an advanced stage because of its inconspicuous location. Endoscopy is the main diagnostic method. Macroscopic appearance is typically intraluminal mass, stenosing, with focal ulceration and contact bleeding. Lesion is pigmented in $85 \%$. Amelanotic forms make the diagnosis even more difficult.

Genomic studies identified several mutations leading to oncogenic transformation of melanocytes. The most

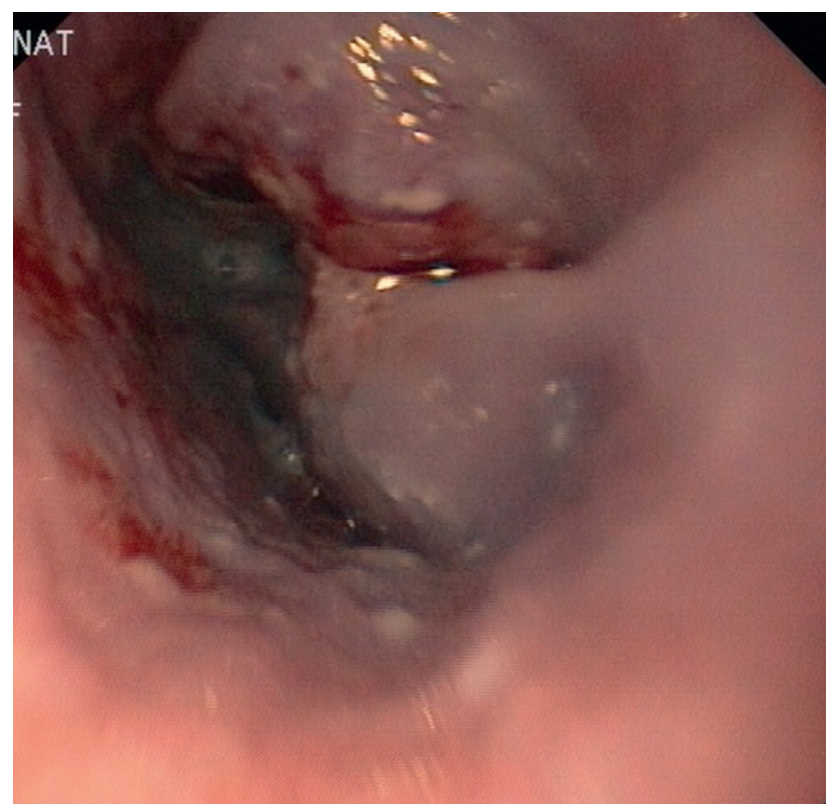

Fig. 1. Endoluminal prominent exulcerated almost circular tumor of esophagus in $30-41 \mathrm{~cm}$ from incisors, fragile, with contact bleeding.

relevant molecular pathways are BRAF ( $60 \%$ of melanomas), N-RAS (15\% of melanomas) and c-Kit (20-30\% of melanomas) (ref., ${ }^{5,6}$ ). Indeed, the treatment landscape is rapidly changing with targeted therapies. Melanomas with KIT mutation are preferentially found on mucosa $(21 \%)$, on sites receiving chronic sun damage (17\%), and on acral skin $(11 \%)$. In contrast, these sites have a very low incidence of $B R A F$ mutation. KIT and BRAF mutations are mutually exclusive ${ }^{6}$.

Masitinib is an oral tyrosine kinase inhibitor that in vitro has greater activity and selectivity than imatinib against both wild-type KIT receptor and its mutated form in the juxtamembrane region ${ }^{7,8}$. Mutations of KIT are expressed in canine mast cell tumors where masitinib has already proven its therapeutic benefit ${ }^{9}$. KIT mutations are also present in gastrointestinal stromal tumors (GIST) where masitinib is currently in comparative phase 3 studies against imatinib and sunitinib in first and second-line treatment settings. Masitinib's activity in imatinib-naïve GIST has previously been reported as sustainable and well-tolerated, and also in imatinib-resistant GIST with less toxicity reported as compared with sunitinib ${ }^{10}$. Masitinib indications are supposed to be much wider, as have documented very hopeful results published recently in indication of pancreatic cancer ${ }^{11}$. Hence, there is strong medical plausibility that masitinib will have a therapeutic benefit in advanced melanoma patients harboring a mutation in the juxtamembrane domain of KIT.

\section{CONCLUSION}

This case report provides a glimpse at the potential therapeutic benefit of masitinib in advanced melanoma carrying mutation in the juxtamembrane domain of KIT. Results from the ongoing phase 3 study (AB08026) will 
therefore be of great interest. It also raises the question of masitinib-treatment beyond progression in this indication under circumstances of overall beneficial response as was the situation in this case despite progression of brain metastases.

Additionally, this case report is also noteworthy due to treatment response observed in brain lesions. It is considered that masitinib's main mode of action in CNS is through its action against mast cell activity thereby reducing the CNS pool of cytokines and chemokines, which act as important promoters of tumorigenesis in a paracrine fashion. Our current observations strongly suggest that under circumstances of brain-blood barrier (BBB) disruption by intracranial metastases masitinib does in fact accumulate in the CNS to exert also direct inhibitory action. This has possible ramifications for masitinib treatment of intracranial neoplasm such as glioblastoma or GIST brain metastases. Masitinib is also being developed for non-oncology indications such as Alzheimer's disease and the prospect that it may be able to cross a disrupted $\mathrm{BBB}$ is very interesting because it brings into play certain additional mechanisms of action.

To the authors' knowledge, this is the first report of brain metastases responding to masitinib.

Author contributions: JP: manuscript writing, literature search, study cooperation, patient care; SL: patient referral, patient care, final approval; JK, JG: study cooperation, patient care; ZP, RK: endoscopy, diagnosis, patient care; BN: histopathology, diagnosis; AM, JA, CM, PD, $\mathrm{OH}$ : study cooperation, masitinib clinical development. Conflict of interest statement: Masitinib is under clinical development by the study sponsor, AB Science (Paris, France). AM, JA and CM are an employees and/or shareholders of the study sponsor. $\mathrm{PD}$ and $\mathrm{OH}$ are consultants and shareholders of the study sponsor. All remaining authors have declared no conflicts of interest.

\section{REFERENCES}

1. Korn EL, Liu PY, Lee SJ, Chapman JA, Niedzwiecki D, Suman VJ, Moon J, Sondak VK, Atkins MB, Eisenhauer EA, Parulekar W, Markovic SN, Saxman S, Kirkwood JM. Meta-analysis of phase II cooperative group trials in metastatic stage IV melanoma to determine progressionfree and overall survival benchmarks for future phase II trials. J Clin Oncol 2008;26(4):527-34.

2. Cote TR, Sobin LH. Primary melanomas of the esophagus and anorectum: epidemiologic comparison with melanoma of the skin. Melanoma Res 2009;19:58-60.

3. Sanchez AA, Wu TT, Prieto VG, Rashid A, Hamilton SR, Wang H. Comparison of primary and metastatic malignant melanoma of the esophagus: clinicopathologic review of 10 cases. Arch Pathol Lab Med 2008;132:1623-9.

4. Chang AE, Karnell LH, Menck HR. The National Cancer Data Base report on cutaneous and noncutaneous melanoma: a summary of 84,836 cases from the past decade. The American College of Surgeons Commission on Cancer and the American Cancer Society. Cancer 1998;83:1664-78.

5. Viros A, Fridlyand J, Bauer J, Lasithiotakis K, Garbe C, Pinkel D, Bastian BC. Improving melanoma classification by integrating genetic and morphologic features. PLoS Med 2008;3;5(6):941-56.

6. Curtin JA, Busam K, Pinkel D, Bastian BC. Somatic activation of KIT in distinct subtypes of melanoma. J Clin Oncol 2006;24:4340-6.

7. Dubreuil P, Létard S, Ciufolini MA, Mansfield CD, Moussy A, Hermine $O$ et al. Masitinib (AB1010), a potent and selective tyrosine kinase inhibitor targeting KIT. PLOS ONE 2009;4(9):2-12.

8. Davis MI, Hunt JP, Herrgard S, Ciceri P, Wodicka LM, Pallares G, Hocker M, Treiber DK, Zarrinkar PP. Comprehensive analysis of kinase inhibitor selectivity. Nat Biotechnol 2011;29(11):1046-51.

9. Hahn KA, Legendre AM, Shaw NG, Phillips B, Ogilvie GK, Prescott DM, Atwater SW, Carreras JK, Lana SE, Ladue T, Rusk A, Kinet JP, Dubreuil $P$, Moussy A, Hermine $O$ et al. Evaluation of 12- and 24-month survival rates after treatment with masitinib in dogs with nonresectable mast cell tumors. Am J Vet Res 2010;71:1354-61.

10. Adenis A, Blay J-Y, Bui-Nguyen B, Bouché O, Bertucci F, Isambert N, Bompas E, Chaigneau L, Domont J, Ray-Coquard I, Blésius A, Van Tine BA, Bulusu VR, Dubreuil P, Mansfield CD, Acin Y, Moussy A, Hermine $\mathrm{O}$, Le Cesne A. Masitinib in advanced gastrointestinal stromal tumor (GIST) after failure of imatinib: A randomized controlled open-label trial. Ann Oncol 2014;25:1762-9.

11. Deplanque G, Demarchi M, Hebbar M, Flynn P, Melichar B, Atkins J, Nowara E, Moyé L, Piquemal D, Ritter D, Dubreuil P, Mansfield CD, Acin Y, Moussy A, Hermine O, Hammel P. A randomized, placebo-controlled phase III trial of masitinib plus gemcitabine in the treatment of advanced pancreatic cancer. Ann Oncol 2015;26(6):1194-200. doi: 10.1093/annonc/mdv133. 